\title{
Can Lightcone Fluctuations be probed with Cosmological Backgrounds?
}

\author{
David Polarski*, Philippe Roche ${ }^{\dagger}$ \\ LPMT, Université Montpellier II, \\ 34095 Montpellier Cedex 05, France
}

October 19, 2018

\begin{abstract}
Finding signatures of quantum gravity in cosmological observations is now actively pursued both from the theoretical and the experimental side. Recent work has concentrated on finding signatures of light-cone fluctuations in the CMB. Because in inflationary scenarios a Gravitational Wave Background (GWB) is always emitted much before the CMB, we can ask, in the hypothesis where this GWB could be observed, what is the imprint of light cone fluctuations on this GWB. We show that due to the flat nature of the GWB spectrum, the effect of lightcone fluctuations are negligible.
\end{abstract}

PACS Numbers: 04.60.-m, 98.80.Cq

Keywords: Quantum Gravity, Inflation, Gravitational Wave Background

*email:polarski@lpta.univ-montp2.fr

$\dagger$ email:roche@lpta.univ-montp2.fr 


\section{Introduction}

Finding signatures of quantum gravity effects in cosmological observations is a subject which is currently explored from the theoretical and the experimental side [1. The existing approaches to quantum gravity, although still in a stage of construction (superstrings, loop quantum gravity), give hints on how quantum gravity effects could be detectable. Possible quantum gravity effects that have been investigated, to name a few, are:

-modification of dispersion laws of propagating particles, through violation of Lorentz invariance (this possibility includes as well birefringence properties of the vacuum). See 2 for a good review of experimental constraints on these effects.

-lightcone fluctuations which could have an imprint through distorsions of the spectrum of observed sources [3].

We will investigate here the second possibility which has been studied in the context of the blackbody Cosmological Microwave Background (CMB) spectrum in [3]. We will review their work and discuss its application to the inflationary primordial Gravitational Wave Background (GWB).

Recent observations of the CMB anisotropy on large and small angular scales are in impressive agreement with the inflationary paradigm, and even with its simplest variant. Indeed, the latest data released by WMAP [4 are in impressive agreement with a flat universe, a scale-invariant primordial spectrum of adiabatic fluctuations obeying gaussian statistics. In the inflationary paradigm, the production of fluctuations observed nowadays in the Universe originates from a genuine quantum process in the Early Universe. The simultaneous generation of a primordial GWB of quantum origin is another fundamental consequence of inflation. The experimental discovery of this coherent GWB spanning about 28 orders of magnitude in frequencies would be a decisive hint at the existence of an inflationary stage [5].

The generation of quantum fluctuations could have taken place at energy scales as high as the GUT scale $\sim 10^{16} \mathrm{GeV}$ but lower scales are certainly possible and

perhaps even more probable. This is far from the Planck scale $\sim 10^{19} \mathrm{GeV}$, the scale at which quantum gravity effects are essential. Therefore if quantum gravity can have an imprint on the GWB observed today, it is not likely to arise through the emission spectrum of the primordial gravitons, but rather through the propagation of these primordial gravitons towards us. As the primordial gravitons are emitted before the CMB photons, actually close to the Big-bang itself, such effects due to their propagation must be at least as large as for CMB photons.

In our work we will discuss the effect of lightcone fluctuations through induced distortions of the primordial GWB. As we will show, even with conservative assumptions, requiring that this effect is detectable leads us to the inconsistent requirement that the inflation scale is of order the Planck scale. This shows that 
quantum gravity effects in the inflationary perturbations due to lightcone fluctuations are basically negligible, well below the threshold detection and do not affect the standard predictions.

\section{Low Energy imprints of Quantum Gravity.}

In this section we recall two notions which have been discussed in the quantum gravity litterature: lightcone fluctuations and the amplification exponent.

The formulation of lightcone fluctuations is background dependent and can be expressed as follows: let $g_{\mu \nu}^{0}$ be a background metric and denote by $h_{\mu \nu}$ the quantum fluctuation around $g_{\mu \nu}^{0}$. The full metric is $g_{\mu \nu}=g_{\mu \nu}^{0}+h_{\mu \nu}$, therefore the local lightcone experiences quantum fluctuations and the propagation of massive and lightlike particles on this space can in principle be affected. The central question is therefore to understand whether these fluctuations affect the low energy physics phenomenology.

Assume that you have two spacetime events $x, y$ and denote by $\sigma(x, y)$ one half of the squared geodesic distance between these points. The notion of lightcone fluctuation leads to the fact that $\sigma(x, y)$ is an operator whose mean value the classical value in the background metric - is $\sigma_{0}(x, y)$ and has an uncertainty $\Delta \sigma(x, y)$. These lightcone fluctuations can in principle be observed: the time $t$ taken by light pulses to travel between a source and a detector separated by a physical distance $l$ has a quantum uncertainty $\Delta t$.

Ensuing central questions are therefore:

1-Is the operator $\sigma(x, y)$ well defined in a theory of quantum gravity?

2-What are the situations where $\Delta t \neq 0$ ? How does it scale with $l$ in those cases?

3 -Is this notion related to the modification of dispersion relations and does $\Delta t$ depend on the nature of the particles and on their energy?

The answers to these questions depend of course on the theoretical framework that one is using for discussing quantum gravity effects.

Question 1 is a central question of conceptual importance but is very poorly understood in the quantum regime. Even in classical general relativity $\sigma(x, y)$ is not an observable (i.e invariant under diffeomorphisms) and one needs to couple general relativity to matter field in order to build true observables [ $[$ ]

We will not dwell further into this very interesting topic but rather state the results that have been obtained perturbatively in $h_{\mu \nu}$ for various backgrounds.

Writing $\sigma=\sigma_{0}+\sigma_{1}+O\left(h_{\mu \nu}^{2}\right)$, where $\sigma_{1}$ is linear in $h_{\mu \nu}$, it can be shown that $\Delta t=\frac{\sqrt{\left\langle\sigma_{1}^{2}\right\rangle}}{l}$ where $l$ is the spatial distance between the two points. In Minkowski space, with one spatial direction compactified on a circle of radius $R$, it was shown 
in [7] that

$$
\Delta t=C t_{P} \sqrt{l / R}
$$

where the spatial points $p, q$ are aligned in a non compactified direction, $l$ is large and $t_{P}$ denotes the Planck time. This can be seen as a refined Casimir effect which disappears in the decompatification limit: there are no lightcone fluctuations at first order in $h_{\mu \nu}$ on a Minkowski background.

Question 2 is related to the notion of amplification exponent which is discussed in [8]. Consider a rod, in a quantum theory of gravity its length is an operator with expectation value $l$ and a quantum uncertainty $\Delta l$. One says that $\Delta l$ satisfies an amplification law with amplification exponent $\alpha$ if the following relation holds true for large $l$ :

$$
\Delta l=C l\left(l_{P} / l\right)^{\alpha} \quad 0<\alpha \leq 1,
$$

where $C$ is a constant of order unity while $l_{P}$ denotes the Planck length. If the amplification law applies, then we would obtain

$$
\Delta t=C t_{P}\left(l / l_{P}\right)^{1-\alpha} .
$$

If it happened that $\alpha=1$, the effect would be so small that it would prevent any attempt to detect lightcone fluctuations. The author of [8] argues using two independent arguments that $\alpha=\frac{2}{3}$. Note that these arguments are far from being convincing and need further confirmation in a quantum gravity theory candidate.

Note that this particular value of $\alpha$ lies on the edge of the range where fluctuations of cosmological distances are detectable.

As we will see later, the value of $\Delta t$ is strongly constrained and for the propagation of photons subject to lightcone fluctuations it satisfies $\Delta t \leq 10^{-14}$. Taking further $l \approx H^{-1}$, we must have $\alpha>0.5$ in order to be detectable.

As for question 3 , in the existing literature on lightcone fluctuations, $\Delta t$ is defined as being the quantum uncertainty of the operator $\sigma(x, y)$. One could measure the fluctuation of the propagation time $\Delta t$ using lightlike particles. However, one should bear in mind that very energetic particles could influence $\Delta t$ in theory of quantum gravity coupled to matter field. In the event that these particles would experience a modification of their dispersion relation, $\Delta t$ could depend on the frequency $\nu$ of the particles used to measure their propagation time. In the present state of our understanding of quantum gravity coupled to matter we can only try a phenomenological approach and look for the possible implications of the dependance of $\Delta t$ on $\nu$.

\section{GWB Distortions from lightcone fluctuations}

As well known, any inflationary scenario produces also a stochastic gravitational waves background [5]. Though its detection would represent a spectacular con- 
firmation of inflation, its direct detection is unfortunately well beyond present technological capability. We will adopt here the point of view that the original GWB spectrum is distorted as a consequence of some stochastic change of frequency due to lightcone fluctuations, a possible quantum gravity effect. We want to investigate when such a distortion is significant.

We will first assume that the time delay, and the corresponding change in frequency, does not depend on the frequency itself (this hypothesis can easily be removed) and further that the stochastic distorted frequency obeys a Gaussian distribution around the undistorted frequency. We follow here the calculations of [3] where it was assumed that the effect originates from (Gaussian) lightcone fluctuations.

We assume that as a consequence of some quantum gravity effects, a monochromatic gravitational wave of frequency $\nu_{0}$ experiences a stochastic distortion obeying a Gaussian distribution with dispersion

$$
\Delta \nu_{0}=\nu_{0}^{2} \Delta t\left(1-\nu_{0} \Delta t\right) \simeq \nu_{0}^{2} \Delta t .
$$

The stochasticity of $\Delta \nu_{0}$ comes from the gaussian random quantity $\Delta t$. Note that we first consider here a fluctuation $\Delta t$ which is independent of the frequency $\nu_{0}$. This assumption can be challenged, as we will briefly discuss below but models were put forward where this assumption holds, the model (3) provides such a example. Note that we deal with models for which the inverse of the quantum metric fluctuations correlation time is much larger than the frequencies $\nu_{0}$ of interest to us 9 .

We consider now any spectral density $F\left(\nu_{0}\right)$. As a result of the stochastic distortion of the frequencies, the new spectral density $\tilde{F}(\nu)$ will be given by

$$
\begin{aligned}
\tilde{F}(\nu) & =\frac{1}{\sqrt{\pi} \Delta \nu_{0}} \int_{0}^{\infty} d \nu_{0} F\left(\nu_{0}\right) e^{-\frac{\left(\nu_{0}-\nu\right)^{2}}{\left(\Delta \nu_{0}\right)^{2}}} \\
& =\frac{1}{\sqrt{\pi}} \int_{-\frac{\nu}{\Delta t}}^{\infty} d z \frac{F(\nu+z \Delta t)}{(\nu+z \Delta t)^{2}} e^{-\frac{z^{2}}{(\nu+z \Delta t)^{4}}}
\end{aligned}
$$

where we have made the change of variable $z=\frac{\nu_{0}-\nu}{\Delta t}$. In order to obtain the expansion in $\Delta t$, we use the following Taylor expansion:

$$
\begin{aligned}
& F[\nu+z \Delta t]=F[\nu]+z \Delta t F^{\prime}[\nu]+\frac{1}{2} z^{2} \Delta t^{2} F^{\prime \prime}[\nu]+o\left(\Delta t^{2}\right) \\
& e^{-\frac{z^{2}}{(\nu+z \Delta t)^{4}}}=e^{-\frac{z^{2}}{\nu^{4}}}\left(1+\frac{4 z^{3} \Delta t}{\nu^{5}}-10 \frac{\Delta t^{2} z^{4}}{\nu^{6}}+8 \frac{z^{6} \Delta t^{2}}{\nu^{10}}\right) .
\end{aligned}
$$

Extending the integration on $z$ in (66) up to $-\infty$ will modify it by a term of the type $e^{-\frac{1}{\nu 2 \Delta t^{2}}}$ which is negligible. In order to compute the expansion in $\Delta t$ one is 
left with Gaussian integrals. The linear term vanishes by parity, so the calculation gives the result:

$$
\delta F \equiv \tilde{F}(\nu)-F(\nu)=F_{2}(\nu) \Delta t^{2}
$$

with

$$
F_{2}(\nu)=3 \nu^{2} F(\nu)+2 \nu^{3} F^{\prime}(\nu)+\frac{\nu^{4}}{4} F^{\prime \prime}(\nu) .
$$

Therefore the fractional distortion of the spectral density $F$ is finally given by

$$
\begin{aligned}
f_{2}(\nu) & =\frac{\tilde{F}(\nu)-F(\nu)}{F(\nu)} \equiv \frac{F_{2}(\nu)}{F(\nu)} \Delta t^{2} \\
& =(\Delta t \nu)^{2}\left[3+2 \nu \frac{F^{\prime}(\nu)}{F(\nu)}+\frac{\nu^{2}}{4} \frac{F^{\prime \prime}(\nu)}{F(\nu)}\right] .
\end{aligned}
$$

We note that all the terms inside the brackets on the l.h.s. of (12) are homogeneous. Therefore, for any spectral density which is a powerlaw in $\nu$,

$$
F(\nu) \propto \nu^{\alpha} \quad \alpha=\text { constant }
$$

the corresponding relative distortion $f_{2}(\nu)$ will be a multiple of $(\Delta t \nu)^{2}$, that is

$$
f_{2}(\nu)=\frac{\nu^{2} \Delta t^{2}}{4}\left[\alpha^{2}+7 \alpha+12\right]
$$

This computation can be easily extended to the case where $\Delta t$ depends on the frequency. Indeed we can still make the change of variable $z=\frac{\nu_{0}-\nu}{\Delta t\left(\nu_{0}\right)}$ which leaves the equation (6) unchanged. We therefore have $z=\frac{\nu_{0}-\nu}{\Delta t\left(\nu_{0}\right)} \simeq \frac{\nu_{0}-\nu}{\Delta t(\nu)}$. As a result we still obtain the equation (12) with $\Delta t$ changed in $\Delta t(\nu)$.

Let us consider the concrete case of the primordial GWB of inflationary origin. For example, one might be interested in the central quantity

$$
\Omega_{G W}=\frac{1}{\rho_{c r}} \frac{\partial \rho_{G W}}{\partial \ln k},
$$

where the wavenumber $k$ is defined as $k \equiv \frac{2 \pi}{c} \nu$. For a class of single-field slow-roll inflationary models, $\Omega_{G W}$ is of the type

$$
\Omega_{G W} \propto k^{n_{T}}
$$

where the tensorial spectral index $n_{T}<0$ is constant and rather small (see below), hence $\Omega_{G W}(\nu)$ is a weakly decreasing function. In that case, (14) can be applied and one finds for the relative distortion of $\Omega_{G W}$

$$
\frac{\delta \Omega_{G W}}{\Omega_{G W}}=\frac{\nu^{2} \Delta t^{2}}{4}\left[n_{T}^{2}+7 n_{T}+12\right] .
$$


The absolute distortion $\delta \Omega_{G W}$ satisfies

$$
\delta \Omega_{G W} \propto \nu^{2+n_{T}} \Delta t^{2} .
$$

Hence, we see that both the fractional and the absolute distortions of $\Omega_{G W}$ grow with $\nu$. Recent $\mathrm{CMB}$ data [4] indicate that the primordial spectrum is nearly scale-invariant, $n_{s} \approx 1$. For slow-roll models with constant spectral indices $n_{s}$, $n_{T}=n_{s}-1$, the tensorial index $n_{T}$ is constrained by observations to be very small, $n_{T} \approx 0$, this is what is meant by a flat GW spectrum. For the fiducial case of a pure scale-invariant spectrum $\left(n_{s}=1\right)$ and $n_{T}=0$ we obtain

$$
\frac{\delta \Omega_{G W}}{\Omega_{G W}}=3(\nu \Delta t)^{2}
$$

while $\Omega_{G W}$ is constant.

Note that we have for the spectral density $F_{G W}(\nu) \equiv \frac{d \rho_{G W}}{d \nu} \propto \nu^{n_{T}-1}$

$$
\begin{aligned}
f_{2}(\nu) & =\frac{\nu^{2} \Delta t^{2}}{4}\left[\left(n_{T}^{2}+5 n_{T}+6\right]\right. \\
\delta F_{G W} & \propto \nu^{1+n_{T}} \Delta t^{2} .
\end{aligned}
$$

As we see from (17), (20), the relative distortion for both quantities $\Omega_{G W}, F_{G W}$ is proportional to the quantity $(\nu \Delta t)^{2}$.

One can also consider slow-roll models with running tensorial spectral index $n_{T}(k)$ but this will introduce no essential change, $f_{2}(\nu)$ will be even smaller. This is because $n_{T}$ is negative and its running is towards larger, i.e. less negative values. It is sufficiently illustrative for our purposes to consider a pure scale-invariant spectrum of density perturbations, $n_{s}=1$. The following result is then obtained instead of (17) after a straightforward calculation

$$
\frac{\delta \Omega_{G W}}{\Omega_{G W}}=(\nu \Delta t)^{2}\left[3+\frac{7}{4} n_{T, H}\left(1-n_{T, H} \ln \frac{\nu}{\nu_{H}}\right)^{-1}+\frac{1}{2} n_{T, H}^{2}\left(1-n_{T, H} \ln \frac{\nu}{\nu_{H}}\right)^{-2}\right],
$$

where $n_{T, H}$ is the index at, and $\nu_{H}$ the frequency associated to, the present Hubble radius. We note that (22) agrees with (17) when $n_{T, H}=0$ because in this model there is no running when $n_{T}=0$. For the highest GWB frequencies we have $\frac{\nu}{\nu_{H}} \approx 10^{28}$.

Observation of the distortion is optimal when both fractional and absolute distortions are maximized, which does not have to take place for the same frequencies. In addition, the observability is constrained by the range of frequencies where the background can be detected at all, whether slightly distorted or not, certainly a crucial constraint for the GWB. From (17/22), we see immediately that the observability of the GWB distortion requires that $(\nu \Delta t)$ is of order one, say 
$10 \%$. As $\Delta t$ is exceedingly small, only very high frequencies $\nu \sim \Delta t^{-1}$ might display a potentially observable distortion. Unfortunately, at very high frequencies a direct detection is problematic at the present time.

It is unlikely that the inflationary GWB could be detected in the near future. Therefore it is impossible at the present time to put experimental constraints on the effect of lightcone fluctuations on the propagation of primordial gravitons. Fortunately we have one cosmological background, namely the Cosmic Microwave Background (CMB), for which we have stringent constraints on possible distortions. Therefore, if we assume the existence of distortions generated by lightcone fluctuations as modeled above, we can set an upper bound on $\Delta t$ using (91012) as explained in [3]. The accurately measured CMB blackbody spectrum constrains the relative distortion to be at most of the order allowed by the FIRAS (COBE) data. Taking for the undistorted distribution $F_{C M B}(\nu)$

$$
F_{C M B}(\nu) \equiv \frac{d \rho_{C M B}}{d \nu}=\frac{8 \pi h}{c^{3}} \frac{\nu^{3}}{e^{\frac{h \nu}{k_{B} T}}-1},
$$

(we have put back in (23) the velocity of light $c$ ) a blackbody radiation distribution with temperature $T \simeq 2.725 \mathrm{~K}$, one can compute its distortion due to lightcone fluctuations using the expressions (91012). As could be expected, the maximal distortion will be for frequencies close to the peak $\nu_{\max }$ of the CMB blackbody spectrum, i.e. for

$$
\nu \approx \nu_{\max }=1.60 \times 10^{11} \mathrm{~Hz}
$$

or equivalently $x \equiv \frac{h \nu}{k_{B} T} \approx x_{\max }=2.82$, the variable introduced in [3]. Note that for the CMB, the fractional distortion $f_{2, C M B}$ satisfies

$$
f_{2, C M B} \propto \Delta t^{2} \nu^{4} \quad \text { for } \nu \rightarrow \infty,
$$

and grows for large frequencies. However, due to the exponential decrease of the blackbody spectrum, the absolute distortion is maximal near $\nu_{\max }$ and tends to zero for large frequencies. This is in contrast with the properties of the quantity $F_{G W}(\nu)$ for which both absolute and relative distortions grow for large frequencies.

The observed blackbody spectrum of the CMB allow for a relative distortion of order $\sim 10^{-5}$ near the peak of the spectrum. Plugging in the numbers, the quantity $\Delta t$ for photons emitted by the CMB is then found to satisfy the bound

$$
\Delta t \leq 10^{-14} \mathrm{~s} .
$$

Though the primordial inflationary gravitational wave background is probably undetectable in the foreseeable future (for technological reasons), still something interesting can be learnt from (20). With the optimistic assumption $\Delta t=10^{-14}$ for primordial gravitons, one would need frequencies as high as $\nu \sim 10^{13} \mathrm{~Hz}$ in 
order for (1722) to be non negligible. This requires in turn an inflationary scale that is of order the Planck mass $M_{p}$ !

Indeed, the highest frequencies of the stochastic gravitational wave background are obtained for those gravitational waves (tensor perturbations) leaving the Hubble radius ("horizon") at the end of inflation. Gravitational waves of the size of the present Hubble radius left the Hubble radius (during inflation) $N$ e-folds before the end of the inflationary stage. Let us consider two inflationary stages with two different energy scales $\Lambda_{1}$ and $\Lambda_{2}\left(H^{2} \propto \Lambda^{4}\right)$. Then it is not hard to deduce the following relation

$$
e^{N_{2}} \simeq \frac{\Lambda_{2}}{\Lambda_{1}} e^{N_{1}}
$$

Even for an inflationary scale $\Lambda_{1}$ of order the GUT scale, $\Lambda_{1} \sim 10^{16} \mathrm{Gev}, N_{1} \sim 65$ and the highest frequencies are $\nu_{\max } \sim 10^{10} \mathrm{~Hz}$. For inflation at lower scales, the highest frequencies will be lower. In order to get frequencies as high as $10^{13} \mathrm{~Hz}$ one needs a $\Lambda$ of order the Planck scale! It is interesting that the upper bound $\Delta t \leq$ $10^{-14}$ s coming from CMB data, totally independently of any model put forward for the generation of the primordial perturbations, implies that the inflationary scale should be close to the Planck scale in order for (1722) to be non negligible.

In the inflationary framework, the spectrum near the highest frequencies requires a careful treatment [11. Indeed, these modes were barely outside the Hubble radius ("horizon"). This is in contrast the modes on cosmological scales which were outside the Hubble radius during most of the universe expansion. It is this all important property that leads to the effective quantum to classical transition of all primordial perturbations, including primordial gravitons, on cosmological scales [10]. This will not apply to the high frequency end of the GWB spectrum.

Leaving aside these problems, at the Planck scale (or higher) the dominant quantum gravity effects are anyway expected to be seen on the emission spectrum itself rather than on the propagation time of the gravitons. We conjecture that a phase transition taking place around the end of the inflationary stage and associated with some feature, like a jump in the first or higher order derivatives in the inflaton potential [12] could alleviate the problem. However observing these potential effects would still remain very unlikely. A more encouraging possibility would be that $\Delta t$ is a growing function of $\nu$ : from a fundamental perspective much work needs to be done on the computation of light cone fluctuations from a theory of quantum gravity. A different effect has been studied in the formalism of Loop Quantum Gravity where it has been stated that modification of dispersion relation of the propagating matter (depending on the frequency $\nu$ ) could arise when evaluated in a spin network state of Loop Quantum Gravity. However because these states are not physical in the sense that they do not satisfy the hamiltonian constraint, conclusions drawn from these computations, if not wrong, have to be taken with the greatest care. A non perturbative understanding of lightcone fluc- 
tuations needs to be adressed but as far as their observability in a cosmological background is concerned the conclusion of our work is negative.

\section{References}

[1] S. Sarkar, "Possible Astrophysical probes of quantum gravity", gr-qc/0204092

[2] "Quantum Gravity Phenomenology and Lorentz Violation", Particle Physics and the Universe, Proceedings of the 9th Adriatic Meeting, eds. J. Trampetic and J. Wess (Springer-Verlag, 2004) gr-qc/0404067

[3] R. DiStefano. L. H. Ford, H. Yu and D.J. Fixsen, "Quantum Gravity and Astrophysics: The Microwave Background and Other Thermal Sources", astro-ph/0107001

[4] D. N. Spergel et al., Ap. J. S. 148 (2003) 175, astro-ph/0302209

[5] A. A. Starobinsky, JETP Lett. 30, 682 (1979)

A. A. Starobinsky, "Non-singular model of the Universe with the quantumgravitational de Sitter stage and its observational consequences", in Quantum Gravity, eds. M.A. Markov, P.C. West, Plenum Publ. Co., N.Y., 1984, pp. 103-128.

B. Allen, Proceedings of the Les Houches school on Astrophysical Sources of Gravitational Waves, Eds. J.-A. Marck and J.-P. Lasota, Cambridge University Press, gr-qc/9604033

[6] C. Rovelli, "What is observable in classical and quantum gravity", Class.Quant.Gravity 8, 297 (1991).

[7] H. Yu and L. H. Ford, "Lightcone Fluctuations in flat space times with non trivial topology", Phys. Rev. D60 (1999) 084023, gr-qc/9904082

[8] Y. Jack Ng, "Space Time foam", Int. J. Mod. Phys. D11 (2002) 1585-1590, $\mathrm{gr}-\mathrm{qc} / 0201022$

[9] H. Yu, L. H. Ford, Phys.Lett. B 496 (2000) 107, gr-qc/9907037

[10] D. Polarski, A. A. Starobinsky, Class. Quantum Grav. 13, (1996) 377, gr-qc/9504030

[11] D. Polarski, Int. J. Mod. Phys. D10 (2001) 927, astro-ph/0109388 
[12] A. A. Starobinsky, JETP Lett. 55 (1992) 489

D. Polarski, Phys. Lett. B 458 (1999) 13, gr-qc/9906075

J. Lesgourgues, D. Polarski, Alexei A. Starobinsky, Mon. Not. Roy. Astron.

Soc. 297 (1998) 769, astro-ph/9711139 